\author{
Zhiling Bai \\ Biomechanics and Biomaterials Laboratory, \\ Department of Applied Mechanics, \\ Beijing Institute of Technology, \\ Beijing 100081, China \\ Yewang $\mathrm{Su}^{1}$ \\ State Key Laboratory of Nonlinear Mechanics, \\ Institute of Mechanics, \\ Chinese Academy of Sciences, \\ Beijing 100190, China \\ e-mail: yewangsu@imech.ac.cn

Baohua Ji
Biomechanics and Biomaterials Laboratory,
Department of Applied Mechanics,
Beijing Institute of Technology,
Beijing 100081, China
e-mail: bhji@bit.edu.cn \\ Baohua Ji ${ }^{1}$ \\ e-mail: bhji@bit.edu.cn
}

\section{Buckling Behaviors of Staggered Nanostructure of Biological Materials}

The nanostructure of biological materials is built with hard mineral crystals embedded in soft protein matrix in a staggered manner. The staggered arrangement of the crystals is assumed to be critically important for the stability of the nanostructure. But the mechanism is not fully understood. In this paper, a mechanical model, considering the effects of overlapping ratio between the crystals, i.e., the staggering position, is developed for analyzing the buckling behaviors of the nanostructure. It is found that the buckling strength increases with the overlapping ratio $\lambda$ in the range of $0-1 / 2$ and reaches a peak value at $\lambda=1 / 2$ that is generally adopted by nature's design of the biological materials. The effect of aspect ratio and volume fraction of mineral crystals are further analyzed at various overlapping ratios, and the results are in general consistent with previous studies for the case of $\lambda=1 / 2$. In addition, the lower and upper limits of the buckling strength are obtained. Finally, we show that the contact between mineral tips can significantly enhance the buckling strength of the nanostructure when the aspect ratio of minerals is small. [DOI: 10.1115/1.4032116]

Keywords: biological materials, buckling, TSC model, nanostructure, hierarchical structure

\section{Introduction}

Natural biological materials, such as bone, teeth, and nacre, have attracted much attention due to their exceptional mechanical properties which have provided valuable inspirations to the synthesis of advanced man-made materials [1-3]. The combination of high strength, hardness, and fracture toughness of the materials enables animals to support their weight, move, and flight, cut foods, and protect themselves against the attack outside [4-6]. These superior mechanical properties are attributed to a design of "brick-and-motar" like nanostructure and its extension to larger scales through the structural hierarchy [2,7-9] (see Fig. 1). This nanostructure is composed of hard mineral crystal and soft protein matrix, and the difference in Young's modulus between them is up to 3 order of magnitude $[2,4]$. In particular, the minerals have large aspect ratio and embedded in the protein matrix in a staggered arrangement [7,10-13] (Fig. 1(c)). The so-called tension-shear chain (TSC) model [7], which has been wellaccepted for depicting the nanostructure of biological materials $[2,10,14,15]$, shows that the mineral crystal bears the tensile/compressive load while the protein matrix transfer the load via shear with the help of large aspect ratio of the mineral. However, because of the large aspect ratio of mineral, the nanostructure is susceptible to buckling when the biological materials are under compression.

Compared with the hardness, strength, and fracture toughness $[2,6-8,11,14]$, the buckling behaviors of the nanostructures are much less explored. Ji et al. [16] analyzed the buckling of a single mineral crystal within the nanostructure. In that preliminary study, only a single mineral was allowed to buckle while its neighboring minerals were kept straight in profile. They found a transition of buckling strength from an aspect-ratio-dependent manner to a saturating behavior with a lower limit that is independent of the aspect ratio. Furthermore, $\mathrm{Su}$ et al. [17] studied the buckling

\footnotetext{
${ }^{1}$ Corresponding author.

Contributed by the Applied Mechanics Division of ASME for publication in the Journal OF ApPLIEd MeChanics. Manuscript received November 1, 2015; final manuscript received November 27, 2015; published online December 16, 2015. Editor: Yonggang Huang.
}

behavior of multiple minerals in the nanostructure, considering the coupling of deformation among adjacent minerals. They showed that there are two typical buckling modes-one is antisymmetric mode which occurs at small aspect ratio, and the other one is symmetric mode which occurs at relatively large aspect
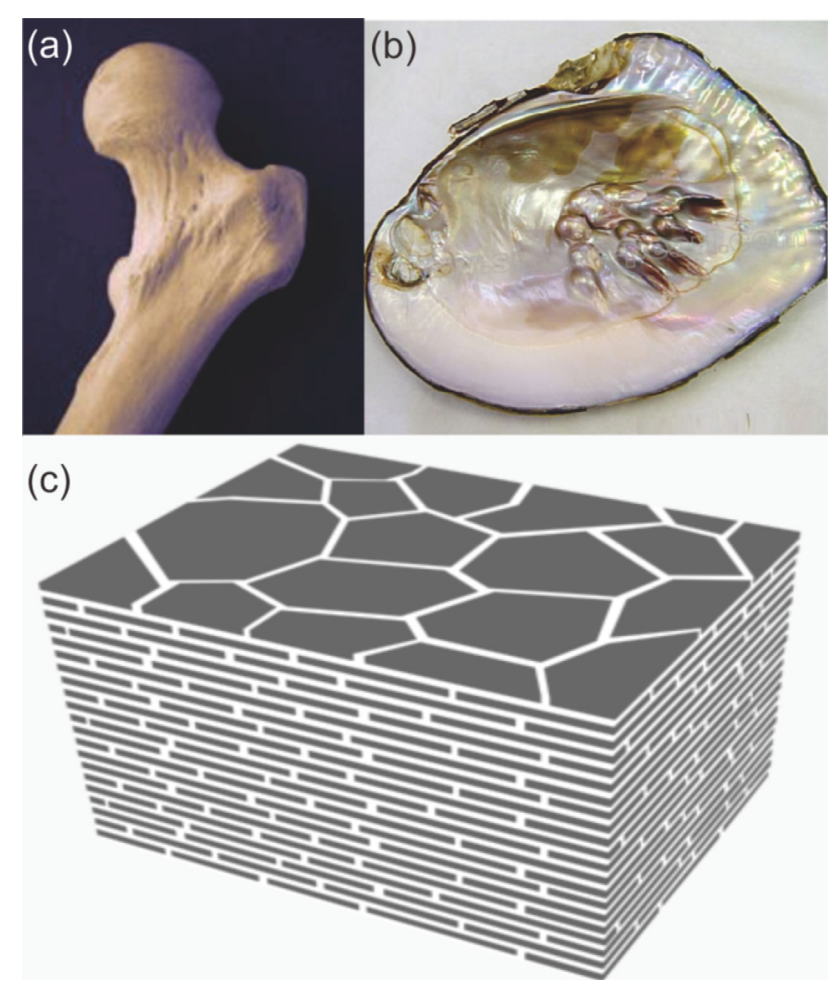

Fig. 1 Typical biological materials and their nanostructures. The images of (a) bone tissue, $(b)$ nacre, and (c) schematic illustration of the nanostructure consisting of hard mineral crystals embedded in soft protein matrix in a staggered manner. 
(a)

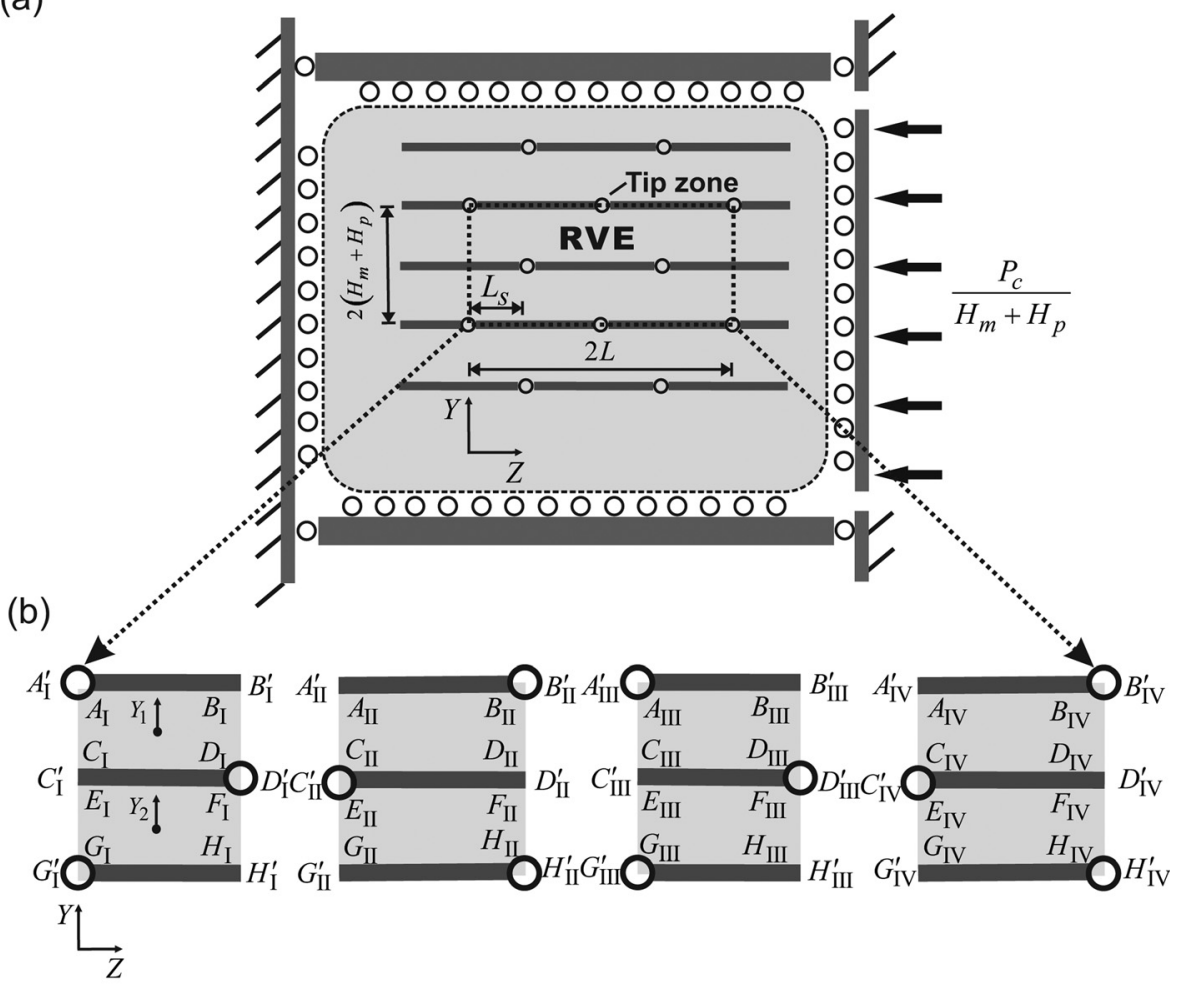

(c)
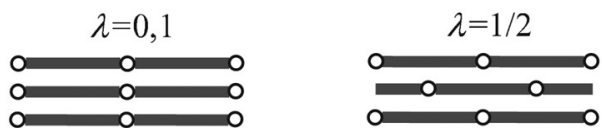

Fig. 2 The model of nanostructure of biological materials under compression. (a) Illustration of the nanostructure where the mineral crystals staggered in the protein matrix with an overlapping length $L_{\mathrm{s}}$. An RVE is line out by a dashed rectangle, (b) partition of the RVE into four parts, denoted by I, II, III, and IV, and (c) two special cases of the staggered arrangement: $\lambda=0,1$ and $\lambda=1 / 2$.

ratio; for very large aspect ratio, the buckling strength of both symmetric and antisymmetric modes approached to that of continuous-fiber reinforced composite given by the Rosen model $[18,19]$. They also showed that the structural hierarchy enhances the buckling strength of biological materials. In addition, they discussed the relationship between local buckling and global buckling which is dependent on the aspect ratio [17].

Despite the advances in understanding the buckling behaviors of the nanostructure made in previous studies, two problems remain unsolved: (1) What is the effect of the staggering position of mineral on the buckling strength? In previous studies, the mineral crystals were assumed in a special staggering with a half overlapping length. (2) How will the contact between mineral tips influence the buckling behaviors? Previously, the possible contact between the adjacent tips of mineral crystals in the longitudinal direction was ignored. However, in practical applications, they may contact with each other when the nanostructure is subjected to compressive load. In this study, we will address these two problems by developing a new theoretical model. The results of this work should advance our knowledge of the buckling behaviors of biological materials and provide guidelines for the design of advanced biomimetic nanocomposites.

\section{The Mechanical Model}

Figure 2(a) shows the nanostructure of biological materials, where the mineral crystals with large aspect ratio are embedded in the protein matrix in a staggered manner. A pressure is applied from the left of the material as the far field load. A representative volume element (RVE) was extracted, which has a length of $2 L$ and a height of $2\left(H_{m}+H_{p}\right)$, where $L$ is the length of the mineral crystal, and $H_{m}$ and $H_{p}$ are the thicknesses of mineral crystal and protein matrix, respectively. Because the RVE is much smaller than the entire nanostructured material, a periodic boundary condition without shear deformation was applied in our buckling analysis, by which the four boundaries of the RVE kept straight before and after the buckling of the minerals (Fig. 2(b)).

The mineral crystals were modeled as slender beams, while the protein matrix was modeled as two-dimensional elastic medium. In previous studies [16,17], the tips between the neighboring minerals were assumed to be free of contact; therefore, the effect of possible contact on the buckling behaviors was neglected. Here, we allowed the tips to contact with each other when the nanostructure is subjected to compressive load. And the contact at the tip zone is modeled as pinned joint in the buckling analysis.

To consider the effect of the overlapping length between mineral crystals, a normalized overlapping length is defined by the ratio $\lambda=L_{s} / L$, called overlapping ratio (staggering position), where the overlapping length $L_{s}$ changes from 0 to $L$, as shown in Fig. $2(a) . \lambda=0,1$ indicate the extreme conditions that the mineral crystals are perfectly aligned, while $\lambda=1 / 2$ indicates the half overlapping, as shown in Fig. 2(c).

To deal with the staggered alignment of the minerals, the RVE is artificially divided into four parts along the $Z$ direction, i.e., I, II, III, and IV. And along the $Y$ direction, there are three layers of minerals, which are denoted by $A_{i}^{\prime} B_{i}^{\prime}, C_{i}^{\prime} D_{i}^{\prime}$, and $G_{i}^{\prime} H_{i}^{\prime}(i=\mathrm{I}$, II, III, IV, see Fig. 2(b)), respectively, where $G_{i}^{\prime} H_{i}^{\prime}$ is the periodic counterpart of $A_{i}^{\prime} B_{i}^{\prime}$ of the next periodic RVE; therefore, the 
deformation and stress resultants of $G_{i}^{\prime} H_{i}^{\prime}$ are identical to those of $A_{i}^{\prime} B_{i}^{\prime}$. In this regard, we only need to analyze the first two layers, i.e., $A_{i}^{\prime} B_{i}^{\prime}$ and $C_{i}^{\prime} D_{i}^{\prime}$. Because of the imaginary cut, there are three joints for each mineral layer, i.e., $B_{\mathrm{I}}^{\prime}-A_{\mathrm{II}}^{\prime}, B_{\mathrm{II}}^{\prime}-A_{\mathrm{III}}^{\prime}$, and $B_{\mathrm{III}}^{\prime}-A_{\mathrm{IV}}^{\prime}$, in the first layer, where $B_{\mathrm{I}}^{\prime}-A_{\mathrm{II}}^{\prime}$ and $B_{\mathrm{III}}^{\prime}-A_{\mathrm{IV}}^{\prime}$ are the rigid joints, while $B_{\mathrm{II}}^{\prime}-A_{\mathrm{III}}^{\prime}$ is the pinned joint; similarly; $D_{\mathrm{I}}^{\prime}-C_{\mathrm{II}}^{\prime}, D_{\mathrm{II}}^{\prime}-C_{\mathrm{III}}^{\prime}$, and $D_{\mathrm{III}}^{\prime}-C_{\mathrm{IV}}^{\prime}$ are in the second layer, where $D_{\mathrm{I}}^{\prime}-C_{\mathrm{II}}^{\prime}$ and $D_{\mathrm{III}}^{\prime}-C_{\mathrm{IV}}^{\prime}$ are the pinned joints, while $D_{\mathrm{II}}^{\prime}-C_{\mathrm{III}}^{\prime}$ is the rigid joint. The symbols without ('), such as $A_{i}, B_{i}, C_{i}$, and $D_{i}$ are used to denote the protein matrix. For example, $A_{\mathrm{I}} B_{\mathrm{I}} C_{\mathrm{I}} D_{\mathrm{I}}$ denotes the matrix between the mineral parts $A_{\mathrm{I}}^{\prime} B_{\mathrm{I}}^{\prime}$ and $C_{\mathrm{I}}^{\prime} D_{\mathrm{I}}^{\prime}$ (Fig. 2(b)).

\section{Static Analysis of the Nanostructures}

We first did the static analysis of the nanostructure before the buckling analysis. $Z$ indicates the horizontal direction and $Y$ indicates the vertical direction, as shown in Fig. 2(a). Because the size of the tip zone is very small, the neighboring minerals in the $Z$ direction would contact with each other once the pressure load is applied on the nanostructure (Fig. 2(a)). In this case, the axial force in mineral crystals is uniform as

$$
\stackrel{\circ}{t_{3}}=P_{c}
$$

Here, the subscript "o" denotes the value before buckling, and the subscript " 3 " denotes the $Z$ direction. Similarly, subscripts " 1 " and " 2 " denote $X$ and $Y$ directions, respectively. The shear deformation in protein matrix is zero because the axial force in mineral crystals is uniform.

We assume that the tips between the minerals will be in contact under compressive load. In this situation, the compressive property of the nanostructure is different from its tensile property. Because the protein is much softer than mineral crystals, the effective compressive modulus of the nanostructure is given by $E_{c}=$ $E_{m} V_{m}$ according to the rule of mixture, where $E_{m}$ and $V_{m}=$ $H_{m} /\left(H_{m}+H_{p}\right)$ are the Young's modulus and the volume fraction of the mineral crystals, respectively. Let $\bar{E}_{c}=E_{c} / E_{p}$ be the normalized compressive modulus of the nanostructure, where $E_{p}$ is the Young's modulus of protein matrix. Then, we have

$$
\bar{E}_{c}=k V_{m}
$$

where $k=E_{m} / E_{p}$ denotes the ratio of the Young's moduli of mineral crystals and the protein matrix. Under the tensile load, the tips between the minerals were separated. In this case, the TSC model gives the normalized tensile modulus as [10]

$$
\bar{E}_{t}=\frac{k \rho^{2} V_{m}^{2}}{8 k\left(1+v_{p}\right)\left(1-V_{m}\right)+\rho^{2} V_{m}}
$$

where $\rho=L / H_{m}$. For $\rho \rightarrow \infty, \bar{E}_{t}$ asymptotically approaches to $\bar{E}_{c}$. Figure 3 gives the comparison of normalized tensile and compressive moduli of the nanostructure. Note that the compressive modulus is proportional to the volume fraction $V_{m}$, independent of the aspect ratio $\rho$. And, it is the upper bound of the tensile modulus.

\section{Buckling Analysis of the Nanostructures}

For the convenience of analysis, the RVE of the nanostructure is divided into four parts as depicted in Fig. 2(b). These four parts share the same global coordinate $Y$ and $Z$, where the origin of the coordinate is $C_{\mathrm{I}}^{\prime}$. For simplification, in the derivation of the governing equations, the subscripts I, II, III, and IV were omitted. They are applicable to all the four parts. But the boundary conditions are different for these four parts.

4.1 Displacements, Strain, and Stress. Let the incremental displacements of mineral crystals in the $Y$ direction due to buckling be (a)
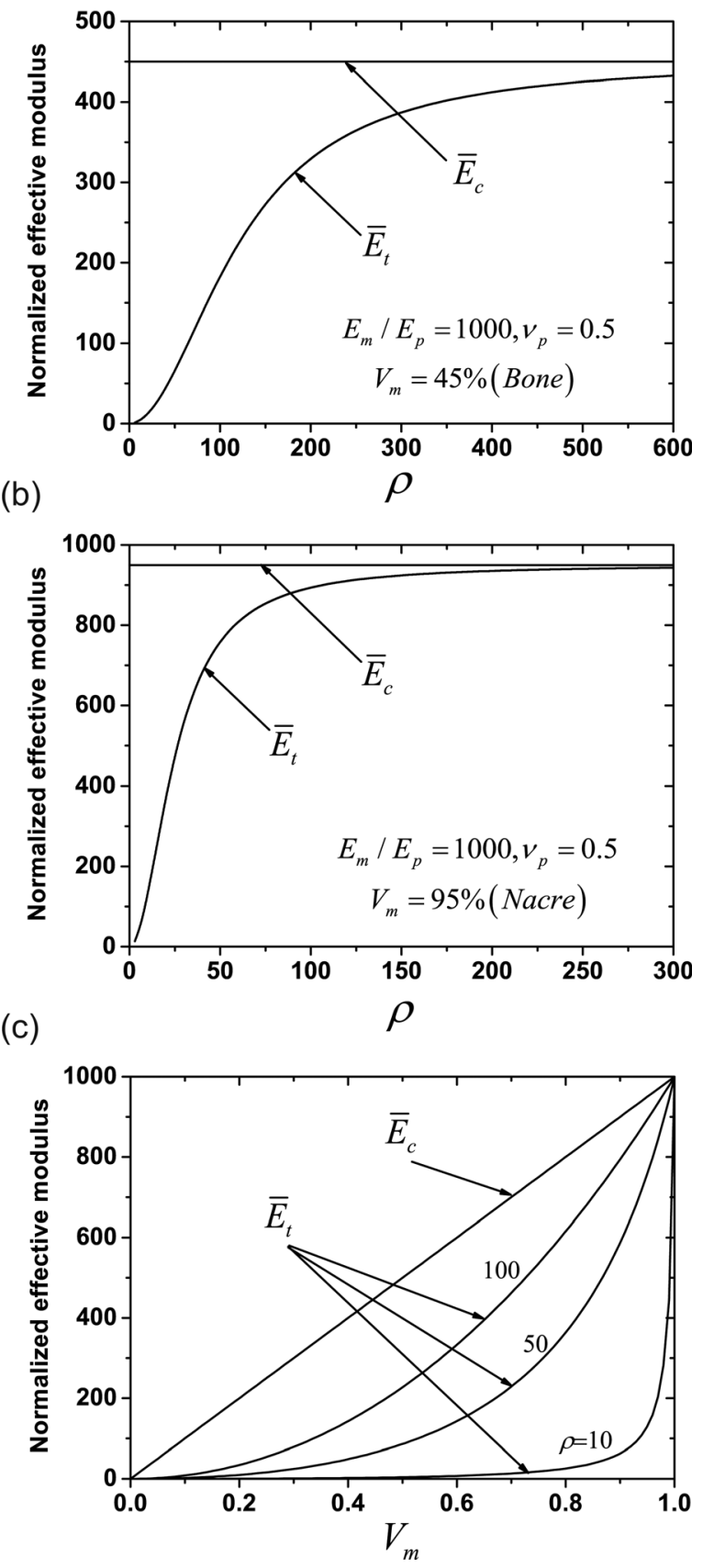

Fig. 3 The normalized tensile and compressive modulus of the nanostructure. The normalized moduli versus aspect ratio for (a) bone $\left(V_{m}=45 \%\right)$, (b) nacre $\left(V_{m}=95 \%\right)$, and (c) the normalized moduli versus the volume fraction of mineral crystals.

$$
\left\{\begin{array}{l}
u_{2}^{A^{\prime} B^{\prime}}(Z)=v_{1}(Z)+C_{1} \\
u_{2}^{C^{\prime} D^{\prime}}(Z)=v_{2}(Z) \\
u_{2}^{G^{\prime} H^{\prime}}(Z)=v_{1}(Z)+C_{2}
\end{array}\right.
$$

where $C_{1}$ and $C_{2}$ are the constant, representing the incremental displacements of the top and bottom minerals at $A_{\mathrm{I}}^{\prime}$ and $G_{\mathrm{I}}^{\prime}$ in the $Y$ direction, respectively, and $v_{1}(Z)$ and $v_{2}(Z)$ are the function for the pure bending deformation. Similarly, let the incremental displacements in the $Z$ direction be 


$$
\left\{\begin{array}{l}
u_{3}^{A^{\prime} B^{\prime}}(Z)=w_{1}(Z)+D_{1} \\
u_{3}^{C^{\prime} D^{\prime}}(Z)=w_{2}(Z) \\
u_{3}^{G^{\prime} H^{\prime}}(Z)=w_{1}(Z)+D_{2}
\end{array}\right.
$$

where $D_{1}$ and $D_{2}$ are the constant, representing the incremental displacements of the top and bottom minerals at $A_{\mathrm{I}}^{\prime}$ and $G_{\mathrm{I}}^{\prime}$ in the $Z$ direction, respectively, and $w_{1}(Z)$ and $w_{2}(Z)$ represent the axial compression/tension deformation.

Now, we derive the displacement of protein matrix. According to the continuity condition at the mineral-protein interface, the incremental displacements for the protein in the $Y$ direction at the interfaces are

$$
\left\{\begin{array}{l}
u_{2}^{A B}(Z)=v_{1}(Z)+C_{1} \\
u_{2}^{C D}(Z)=v_{2}(Z) \\
u_{2}^{E F}(Z)=v_{2}(Z) \\
u_{2}^{G H}(Z)=v_{1}(Z)+C_{2}
\end{array}\right.
$$

In the $Z$ direction, the displacement of the mineral-protein interface is yielded by both the bending and the tensile/compressive of the minerals, which is given by

$$
\left\{\begin{array}{l}
u_{3}^{A B}(Z)=u_{3}^{A^{\prime} B^{\prime}}(Z)+\frac{H_{m}}{2} \frac{d}{d Z} u_{2}^{A^{\prime} B^{\prime}}(Z)=w_{1}(Z)+\frac{H_{m}}{2} v_{1}^{\prime}(Z)+D_{1} \\
u_{3}^{C D}(Z)=u_{3}^{C^{\prime} D^{\prime}}(Z)-\frac{H_{m}}{2} \frac{d}{d Z} u_{2}^{C^{\prime} D^{\prime}(Z)}=w_{2}(Z)-\frac{H_{m}}{2} v_{2}^{\prime}(Z) \\
u_{3}^{E F}(Z)=u_{3}^{C^{\prime} D^{\prime}}(Z)+\frac{H_{m}}{2} \frac{d}{d Z} u_{2}^{C^{\prime} D^{\prime}}(Z)=w_{2}(Z)+\frac{H_{m}}{2} v_{2}^{\prime}(Z) \\
u_{3}^{G H}(Z)=u_{3}^{G^{\prime} H^{\prime}}(Z)-\frac{H_{m}}{2} \frac{d}{d Z} u_{2}^{G^{\prime} H^{\prime}}(Z)=w_{1}(Z)-\frac{H_{m}}{2} v_{1}^{\prime}(Z)+D_{2}
\end{array}\right.
$$

Here ()$^{\prime}=d() / d Z$.

Then, the shear stress in protein matrix can be derived by substituting the geometry relation $\gamma=\left(\partial u_{3} / \partial Y+\partial u_{2} / \partial Z\right)$ into $\tau=G_{p} \gamma$ as

$$
\tau=G_{p}\left(\frac{\partial u_{3}}{\partial Y}+\frac{\partial u_{2}}{\partial Z}\right)
$$

where $G_{p}=E_{p} /\left[2\left(1+\nu_{p}\right)\right]$, and $E_{p}$ and $\nu_{p}$ are the Young's modulus and Poisson's ratio of protein matrix, respectively. We assume that the shear stress in protein matrix is constant along the $Y$ direction as the ratio of thickness to the length of protein layer is small. By the means of average, the shear stress in protein layer can be calculated as

$$
\left\{\begin{array}{l}
\tau^{A D}(Z)=G_{p}\left[\frac{1}{2}\left(\frac{d}{d Z} u_{2}^{A B}(Z)+\frac{d}{d Z} u_{2}^{C D}(Z)\right)+\frac{1}{H_{p}}\left(u_{3}^{A B}(Z)-u_{3}^{C D}(Z)\right)\right] \\
\tau^{E H}(Z)=G_{p}\left[\frac{1}{2}\left(\frac{d}{d Z} u_{2}^{E F}(Z)+\frac{d}{d Z} u_{2}^{G H}(Z)\right)+\frac{1}{H_{p}}\left(u_{3}^{E F}(Z)-u_{3}^{G H}(Z)\right)\right]
\end{array}\right.
$$

where $A D$ and $E H$ denote protein matrix $A B C D$ and $E F G H$ (the superscripts I, II, III, and IV are omitted, see Fig. 2(a)), respectively. According to the equilibrium equation (Fig. 4(a))

$$
\frac{\partial \sigma_{Y}}{\partial Y}+\frac{\partial \tau}{\partial Z}=0
$$

the normal stress $\sigma_{Y}$ along the $Y$ direction is a linear function of $Y$, but with a constant to be determined. Hence, the normal stress $\sigma_{Y}$ in protein matrix is obtained as

$$
\left\{\begin{aligned}
\sigma_{Y}^{A D}(Z)= & G_{p} Y_{1}\left[\frac{1}{2}\left(\frac{d^{2}}{d Z^{2}} u_{2}^{A B}(Z)+\frac{d^{2}}{d Z^{2}} u_{2}^{C D}(Z)\right)+\frac{1}{H_{p}}\left(\frac{d}{d Z} u_{3}^{A B}(Z)-\frac{d}{d Z} u_{3}^{C D}(Z)\right)\right] \\
& +\frac{E_{p}}{1-\nu_{p}{ }^{2}} \frac{u_{2}^{A B}(Z)-u_{2}^{C D}(Z)}{H_{p}} \\
\sigma_{Y}^{E H}(Z)= & G_{p} Y_{2}\left[\frac{1}{2}\left(\frac{d^{2}}{d Z^{2}} u_{2}^{E F}(Z)+\frac{d^{2}}{d Z^{2}} u_{2}^{G H}(Z)\right)+\frac{1}{H_{p}}\left(\frac{d}{d Z} u_{3}^{E F}(Z)-\frac{d}{d Z} u_{3}^{G H}(Z)\right)\right] \\
& +\frac{E_{p}}{1-\nu_{p}^{2}} \frac{u_{2}^{E F}(Z)-u_{2}^{G H}(Z)}{H_{p}}
\end{aligned}\right.
$$


(a)
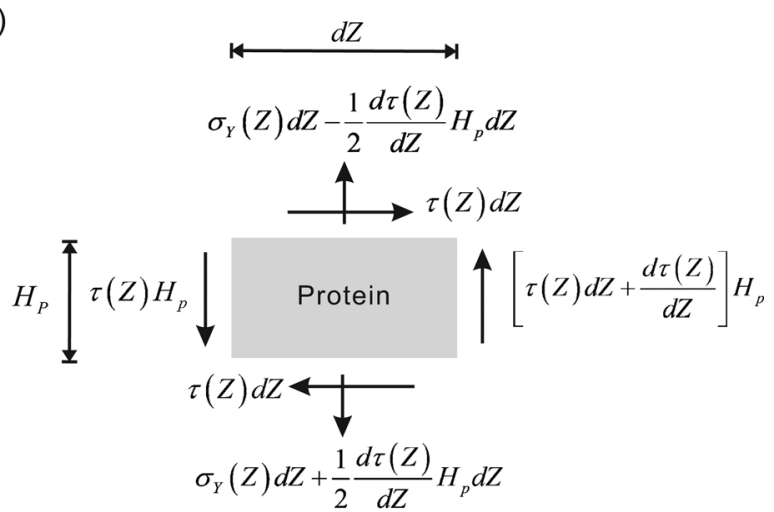

(b)

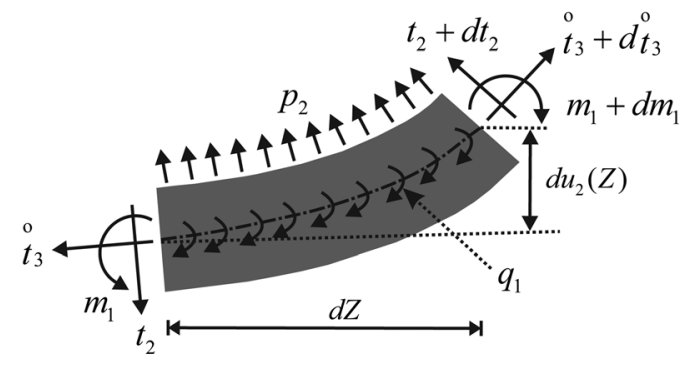

Fig. 4 Free-body diagrams of elements of (a) protein matrix and $(b)$ mineral crystal

where $Y_{1}$ and $Y_{2}$ start from the center of protein matrix $A B C D$ and $E F G H$, respectively, as depicted in Fig. 2(b). With $Y_{1}= \pm H_{p} / 2$ and $Y_{2}= \pm H_{p} / 2$, the stress at the interface $A B, C D, E F$, and $G H$ can be obtained, respectively. And, we have $\sigma_{Y}=\left[E_{p} /(1-\right.$ $\left.\left.\nu_{p}^{2}\right)\right]\left[u_{2}^{A B}(Z)-u_{2}^{C D}(Z)\right] / H_{p}$ at the horizontal center line of protein matrix $A B C D$, and similar result is applied to protein $E F G H$.

4.2 Governing Equations of Minerals. According to the illustration in Fig. 4(b), the equilibrium equation for the mineral modeled by a plane beam is given by

$$
\frac{d^{2} m_{1}}{d Z^{2}}+\frac{d q_{1}}{d Z}+\stackrel{\circ}{t_{3}} \frac{d^{2} u_{2}}{d Z^{2}}+\frac{d t_{3}^{\circ}}{d Z} \frac{d u_{2}}{d Z}+p_{2}=0
$$

where $m_{1}$ is the bending moment; $q_{1}$ and $p_{2}$ are the distributed moment and force, respectively; and $t_{3}$ is the axial force before buckling. According to Eq. (1), $t_{3}=P_{c}$ is a constant. For the mineral crystal $A^{\prime} B^{\prime}$

$$
\begin{aligned}
& m_{1}(Z)=-\frac{E_{m} H_{m}^{3}}{12} v_{1}^{\prime \prime}(Z) \\
& q_{1}(Z)=\frac{H_{m}}{2}\left[\tau^{E H}(Z)+\tau^{A D}(Z)\right] \\
& p_{2}(Z)=\left.\sigma_{Y}^{E H}(Z)\right|_{Y_{2}=-H_{P} / 2}-\left.\sigma_{Y}^{A D}(Z)\right|_{Y_{1}=H_{P} / 2}
\end{aligned}
$$

Similarly, for the mineral crystal $C^{\prime} D^{\prime}$

$$
\begin{aligned}
& m_{1}(Z)=-\frac{E_{m} H_{m}^{3}}{12} v_{2}^{\prime \prime}(Z) \\
& q_{1}(Z)=\frac{H_{m}}{2}\left[\tau^{A D}(Z)+\tau^{E H}(Z)\right] \\
& p_{2}(Z)=\left.\sigma_{Y}^{A D}(Z)\right|_{Y_{2}=-H_{P} / 2}-\left.\sigma_{Y}^{E H}(Z)\right|_{Y_{1}=H_{P} / 2}
\end{aligned}
$$

Substituting Eqs. (13) and (14) into Eq. (12), together with Eqs. (4)-(7), (9), and (11), the governing equation of minerals $A^{\prime} B^{\prime}$ and $C^{\prime} D^{\prime}$ are obtained, respectively, as

$$
\begin{aligned}
-\frac{E_{m} H_{m}^{3}}{12} v_{1}^{(4)}(Z) & +\frac{G_{p}\left(H_{m}+H_{p}\right)^{2}}{2 H_{p}}\left[v_{1}^{\prime \prime}(Z)+v_{2}^{\prime \prime}(Z)\right]+P_{c} v_{1}^{\prime \prime}(Z) \\
& +\frac{2 E_{p}}{H_{p}\left(1-\nu_{p}^{2}\right)}\left[\left(v_{2}(Z)-v_{1}(Z)-\frac{C_{1}+C_{2}}{2}\right]=0\right. \\
-\frac{E_{m} H_{m}^{3}}{12} v_{2}^{(4)}(Z) & +\frac{G_{p} H_{m}\left(H_{m}+H_{p}\right)}{2 H_{p}}\left[v_{1}^{\prime \prime}(Z)+v_{2}^{\prime \prime}(Z)\right] \\
& +P_{c} v_{2}^{\prime \prime}(Z)+\frac{2 E_{p}}{H_{p}\left(1-\nu_{p}^{2}\right)} \\
& \times\left[\left(v_{1}(Z)-v_{2}(Z)+\frac{C_{1}+C_{2}}{2}\right]=0\right.
\end{aligned}
$$

Note that the incremental displacements along the $Z$ direction do not appear in the governing equations because they are of smaller order of magnitude compared with the displacement in the $Y$ direction [20].

To simplify the governing equation, we define

$$
\left\{\begin{array}{l}
f(\xi)=v_{1}(\mathrm{Z})+v_{2}(\mathrm{Z}) \\
g(\xi)=v_{1}(Z)-v_{2}(Z)+\left(C_{1}+C_{2}\right) / 2
\end{array}\right.
$$

where $\xi=Z / L$ denotes the normalized coordinate of $Z$. Thus, Eq. (15) becomes

$$
\left\{\begin{array}{l}
f^{(4)}(\xi)+\alpha^{2} f^{\prime \prime}(\xi)=0 \\
g^{(4)}(\xi)+2 e_{1} g^{\prime \prime}(\xi)+e_{2}^{2} g(\xi)=0
\end{array}\right.
$$

Here, ()$^{(4)}=d^{4}() / d \xi^{4}$ and ()$^{\prime \prime}=d^{2}() / d \xi^{2}$, and

$$
\begin{aligned}
& \alpha^{2}=\frac{12 \rho^{2}}{k}\left(\frac{\bar{\sigma}_{c}}{V_{m}}-\frac{1}{2 V_{m}\left(1-V_{m}\right)\left(1+\nu_{p}\right)}\right), \quad e_{1}=\frac{6 \rho^{2}}{k} \frac{\bar{\sigma}_{c}}{V_{m}}, \\
& e_{2}^{2}=\frac{48 \rho^{4}}{k} \frac{V_{m}}{\left(1-V_{m}\right)\left(1-\nu_{p}^{2}\right)}
\end{aligned}
$$

are dimensionless parameters, and

$$
\bar{\sigma}_{c}=\frac{-P_{c}}{E_{p}\left(H_{m}+H_{p}\right)}
$$

is the normalized critical buckling stress.

Solving the governing equation (17), we have the general solutions as

for

$$
e_{1}<e_{2},\left\{\begin{aligned}
f(\xi)= & G_{5} \sin (\alpha \xi)+G_{6} \cos (\alpha \xi)+G_{7} \xi+G_{8} \\
g(\xi)= & G_{1} \cosh \left(\beta_{1} \xi\right) \cos \left(\beta_{2} \xi\right)+G_{2} \sinh \left(\beta_{1} \xi\right) \cos \left(\beta_{2} \xi\right) \\
& +G_{3} \cosh \left(\beta_{1} \xi\right) \sin \left(\beta_{2} \xi\right)+G_{4} \sinh \left(\beta_{1} \xi\right) \sin \left(\beta_{2} \xi\right)
\end{aligned}\right.
$$

where $\beta_{1}=\sqrt{\left(e_{2}-e_{1}\right) / 2}$ and $\beta_{2}=\sqrt{\left(e_{2}+e_{1}\right) / 2}$. and for

$$
e_{1}>e_{2},\left\{\begin{aligned}
f(\xi)= & G_{5} \sin (\alpha \xi)+G_{6} \cos (\alpha \xi)+G_{7} \xi+G_{8} \\
g(\xi)= & G_{1} \cos \left(\beta_{1} \xi\right)+G_{2} \sin \left(\beta_{1} \xi\right)+G_{3} \cos \left(\beta_{2} \xi\right) \\
& +G_{4} \sin \left(\beta_{2} \xi\right)
\end{aligned}\right.
$$


where $\beta_{1}=\sqrt{e_{1}+\sqrt{e_{1}^{2}-e_{2}^{2}}}$ and $\beta_{2}=\sqrt{e_{1}-\sqrt{e_{1}^{2}-e_{2}^{2}}}$. Substitution of Eqs. (20a) and (20b) into Eq. (16) gives the incremental displacements as

$$
\left\{\begin{array}{l}
v_{1}(\xi)=\frac{f(\xi)+g(\xi)}{2}-\frac{C_{1}+C_{2}}{4} \\
v_{2}(\xi)=\frac{f(\xi)-g(\xi)}{2}+\frac{C_{1}+C_{2}}{4}
\end{array}\right.
$$

Substituting Eq. (21) into Eq. (4), we obtained the displacements of mineral crystals. The constants in the solution can be determined by the continuity and boundary conditions. As there are four parts in the RVE, we have $8 \times 4=32$ constants according to Eq. (20). In addition to constants $C_{1}$ and $C_{2}$ in Eq. (21), we have 34 constants that to be determined.

4.3 Continuity Conditions and Boundary Conditions. The continuity conditions should be applied at the interfaces of two neighboring parts. We define I-II as the interface between parts I and II, and similarly II-III and III-IV the interfaces of parts II and III and parts III and IV, respectively. For example, there are two joints at the I-II interface, $B_{\mathrm{I}}^{\prime} A_{\mathrm{II}}^{\prime}$ and $D_{\mathrm{I}}^{\prime} C_{\mathrm{II}}^{\prime}$ (Fig. $2(b)$ ). $B_{\mathrm{I}}^{\prime} A_{\mathrm{II}}^{\prime}$ is rigid joint, i.e., the displacement $u_{2}$, angle $\theta$, moment $m_{1}$, and shear force $Q$ all should be continuous; But $D_{\mathrm{I}}^{\prime} C_{\mathrm{II}}^{\prime}$ is pinned joint, therefore the continuity of rotation angle does not hold, instead the condition of zero moment should be applied. Therefore, the continuity conditions at the I-II interface are written as

$$
\begin{aligned}
u_{2}^{B_{\mathrm{I}}^{\prime}} & =u_{2}^{A_{\mathrm{II}}^{\prime}}, \quad \theta^{B_{\mathrm{I}}^{\prime}}=\theta^{A_{\mathrm{II}}^{\prime}}, \quad m_{1}^{B_{\mathrm{I}}^{\prime}}=m_{1}^{A_{\mathrm{II}}^{\prime}}, \quad Q^{B_{\mathrm{I}}^{\prime}}=Q^{A_{\mathrm{II}}^{\prime}}, \quad u_{2}^{D_{\mathrm{I}}^{\prime}}=u_{2}^{C_{\mathrm{II}}^{\prime}}, \\
m_{1}^{D_{\mathrm{I}}^{\prime}} & =0, \quad m_{1}^{C_{\mathrm{II}}^{\prime}}=0, \quad Q^{D_{\mathrm{I}}^{\prime}}=Q^{C_{\mathrm{II}}^{\prime}}
\end{aligned}
$$

Similarly, the continuity conditions for the II-III and III-IV interfaces are

$$
\begin{aligned}
& u_{2}^{B_{\mathrm{II}}^{\prime}}=u_{2}^{A_{\mathrm{II}}^{\prime}}, \quad m_{1}^{B_{\mathrm{II}}^{\prime}}=0, \quad m_{1}^{A_{\mathrm{II}}^{\prime}}=0, \quad Q^{B_{\mathrm{II}}^{\prime}}=Q^{A_{\mathrm{III}}^{\prime}}, \quad u_{2}^{D_{\mathrm{II}}^{\prime}}=u_{2}^{C_{\mathrm{II}}^{\prime},} \\
& \theta^{D_{\mathrm{II}}^{\prime}}=\theta^{C_{\mathrm{III}}^{\prime}}, \quad m_{1}^{D_{\mathrm{II}}^{\prime}}=m_{1}^{C_{\mathrm{III}}^{\prime}}, \quad Q^{D_{\mathrm{II}}^{\prime}}=Q^{C_{\mathrm{III}}^{\prime}} \\
& u_{2}^{B_{\mathrm{II}}^{\prime}}=u_{2}^{A_{\mathrm{IV}}^{\prime}}, \quad \theta^{B_{\mathrm{II}}^{\prime}}=\theta^{A_{\mathrm{IV}}^{\prime}}, \quad m_{1}^{B_{\mathrm{II}}^{\prime}}=m_{1}^{A_{\mathrm{IV}}^{\prime}}, \quad Q^{B_{\mathrm{III}}^{\prime}}=Q^{A_{\mathrm{IV}}^{\prime}}, \\
& u_{2}^{D_{\mathrm{III}}^{\prime}}=u_{2}^{C_{\mathrm{III}}^{\prime}}, \quad m_{1}^{D_{\mathrm{III}}^{\prime}}=0, \quad m_{1}^{C_{\mathrm{II}}}=0, \quad Q^{D_{\mathrm{III}}^{\prime}}=Q^{C_{\mathrm{III}}^{\prime}} \quad(22 c)
\end{aligned}
$$

Then, we have $8 \times 3=24$ continuity conditions at the interfaces. Here, $\theta=d u_{2} / d Z$, and the expression of shear forces $Q$ is given in the Appendix.

Regarding the boundary conditions, the constraint of rigid-body motion and the periodic conditions should be applied. To constrain the rigid-body motion of the RVE in the $Y$ direction, we fixed the displacement of $C_{\mathrm{I}}^{\prime}$, i.e., $u_{2}^{C_{\mathrm{I}}^{\prime}}=0$, and let $v_{1}(Z)=0$ at $A_{\mathrm{I}}^{\prime}$. These constraint conditions are expressed as

$$
u_{2}^{C_{1}^{\prime}}=u_{2}^{C_{1}^{\prime} D_{1}}(0)=0 ; \quad u_{2}^{A_{1}}=u_{2}^{A_{1} B_{1}}(0)=C_{1}
$$

On the other hand, the periodic condition requires the continuity of displacement, angle, and moment at the periodic boundary as

$$
\begin{array}{ll}
u_{2}^{A_{1}^{\prime}}=u_{2}^{B_{\mathrm{IV}}^{\prime}}, & m_{1}^{A_{\perp}^{\prime}}=0, \quad m_{1}^{A_{\perp}^{\prime}}=m_{1}^{B_{\mathrm{IV}}^{\prime}}, \quad u_{2}^{C_{\mathrm{I}}^{\prime}}=u_{2}^{D_{\mathrm{IV}}^{\prime}}, \\
\theta^{C_{\mathrm{I}}^{\prime}}=\theta^{D_{\mathrm{IV}}^{\prime}}, & m_{1}^{C_{\mathrm{I}}^{\prime}}=m_{1}^{D_{\mathrm{IV}}^{\prime}}
\end{array}
$$

In addition, the entire nanostructure is force free in the $Y$ direction; therefore, the integral of stress $\sigma_{Y}$ on any horizontal section of the RVE should be zero. For simplification, we apply this condition to the upper and bottom boundary of protein matrix $A B C D$ and $E F G H$ as

$$
\begin{aligned}
\int_{\Omega} \sigma_{Y}^{A_{\mathrm{I}} B_{\mathrm{IV}}}(Z) d Z & =\int_{\Omega} \sigma_{Y}^{C_{\mathrm{I}} D_{\mathrm{IV}}}(Z) d Z=\int_{\Omega} \sigma_{Y}^{E_{\mathrm{I}} F_{\mathrm{IV}}}(Z) d Z \\
& =\int_{\Omega} \sigma_{Y}^{G_{1} H_{\mathrm{IV}}}(Z) d Z=0
\end{aligned}
$$

which yields two conditions as

$$
\begin{aligned}
C_{1}= & C_{2}, \int_{0}^{\lambda} g_{\mathrm{I}}(\xi) d \xi+\int_{\lambda}^{1} g_{\mathrm{II}}(\xi) d \xi+\int_{1}^{1+\lambda} g_{\mathrm{III}}(\xi) d \xi \\
& +\int_{1+\lambda}^{2} g_{\mathrm{IV}}(\xi) d \xi=0
\end{aligned}
$$

Here, $g_{i}(\xi)(i=\mathrm{I}, \mathrm{II}, \mathrm{III}, \mathrm{IV})$ is the solution of Eq. $(17)_{2}$.

Taken together, we now have $8 \times 4+2=34$ conditions/equations, of which the number is exactly equal to that of the constants to be determined.

4.4 Solution of Buckling Strength. With Eqs. (22), (23), and (25), we obtained a system of homogeneous linear equations

$$
M U=\mathbf{0}
$$

from which the buckling strength of the nanostructure, $\bar{\sigma}_{c}$, can be determined. Here, $\boldsymbol{U}$ is a vector consisting of all the unknown constants, including constants $C_{1}$ and $C_{2}$ in Eq. (16) and constants $G_{\mathrm{i}}$ $(i=1-8)$ in Eq. (20) of four parts (I, II, III, and IV); and $\boldsymbol{M}$ is the corresponding coefficient matrix of dimension of $34 \times 34$. The condition of nontrivial solution of $\boldsymbol{U}$ requires the determinant of $\boldsymbol{M}$ to be zero, i.e.,

$$
\operatorname{det}(\boldsymbol{M})=0
$$

Solving Eq. (27) gives the solution of the normalized critical buckling stress $\bar{\sigma}_{c}$. Shooting method is used to solve the equation.

\section{Results and Discussion}

The aim of this study is to examine the effect of staggering of minerals and the contact between mineral tips on the buckling strength of nanostructure. Figure 5 shows the normalized buckling strength, i.e., normalized critical buckling stress, as a function of overlapping ratio (staggering position) for volume fraction $V_{m}=$ $45 \%$ and aspect ratio $\rho=10$. As we can see, the buckling strength increases with the overlapping ratio $\lambda$ in the range of $\lambda \in[0,1 / 2]$, while it decreases in the range of $\lambda \in[1 / 2,1]$, i.e., the plot is symmetric with respect to $\lambda=(1 / 2)$. To understand the dependence of the buckling strength on $\lambda$, we examined the changing of the

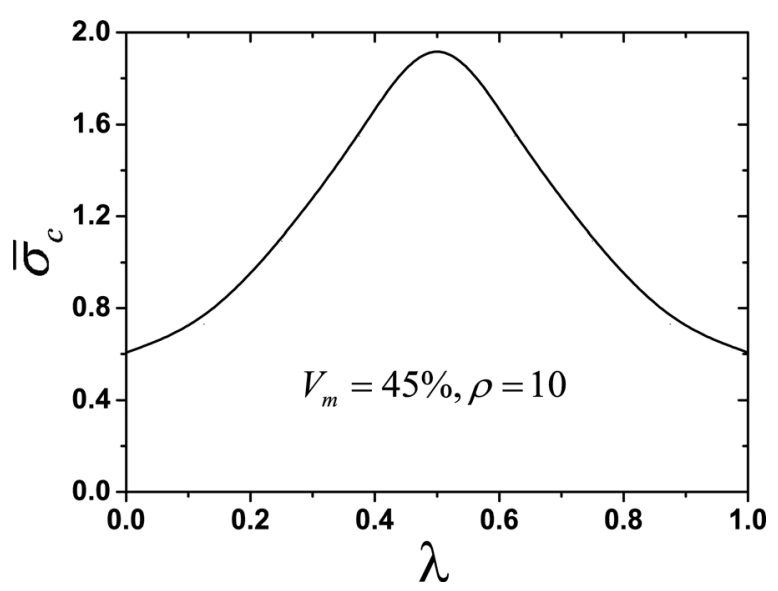

Fig. 5 The effect of overlapping ratio $\lambda$ on the normalized buckling strength of the nanostructure $\bar{\sigma}_{c}$ in the case of $V_{m}=45 \%$ and $\rho=10$ 
buckling mode with $\lambda$. For $\lambda=0$, the mineral performed a rigidbody rotation without bending as depicted in Fig. 6, and the protein matrix had a shear-dominated deformation. The minerals are able to rotate easily via the pinned joints because of no overlapping between neighboring minerals. The buckling strength at this special alignment defines the lower limit of the strength of all buckling modes at different overlapping ratios (see Figs. 5 and 7), which is given by [17]

\begin{tabular}{|c|c|c|c|c|}
\hline$V_{m}=45 \%$ & $\rho=5$ & $\rho=10$ & $\rho=20$ & $\rho=50$ \\
\hline$\lambda=0$ & & & & \\
\hline$\lambda=1 / 8$ & & & & \\
\hline$\lambda=1 / 4$ & & & & \\
\hline$\lambda=3 / 8$ & & & & \\
\hline$\lambda=1 / 2$ & & & & \\
\hline
\end{tabular}

Fig. 6 The predicted buckling modes of the nanostructure changing with the aspect ratio and overlapping ratio

(a)

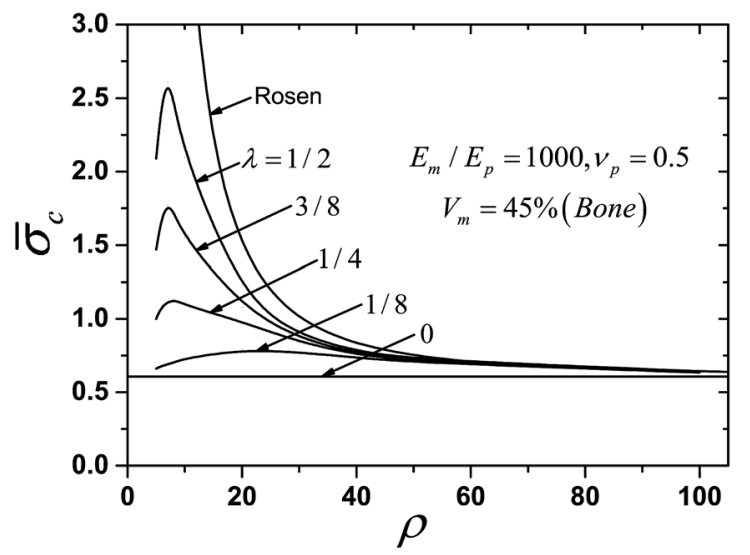

(b)

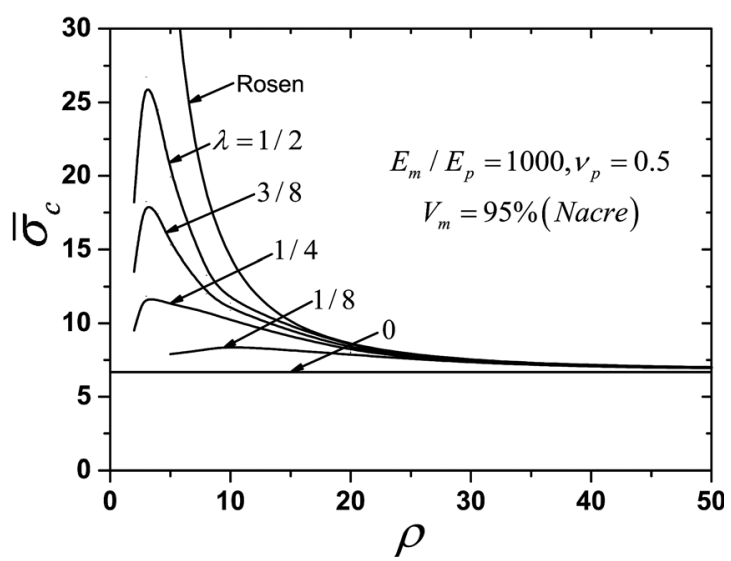

Fig. 7 The effect of overlapping ratio $\lambda$ and aspect ratio $\rho$ on the buckling strength $\bar{\sigma}_{c}$ for $(a)$ bone $\left(V_{m}=45 \%\right)$ and $(b)$ nacre $\left(V_{m}=95 \%\right)$

$$
\bar{\sigma}_{c}^{L}=\frac{1}{2\left(1-V_{m}\right)\left(1+\nu_{p}\right)}
$$

Note that it is independent of the aspect ratio. With the increase of $\lambda$, the rotation of minerals was more and more constrained by their neighboring minerals. Thus, the bending of minerals and tension of protein along the $Y$ direction played more important roles. When the overlapping ratio was increased to $\lambda=1 / 2$, the buckling strength reached its maximum value (Fig. 5), indicating that the staggered arrangement is of critical importance for enhancing the buckling strength of the nanostructure. In fact, $\lambda=1 / 2$ is usually adopted by nature in the design of nanostructures of biological materials.

We also found that the aspect ratio takes critical role in the buckling strength of the nanostructure. Figure 7 illustrates the effect of aspect ratio on buckling strength at different overlapping ratios $\lambda$ for two typical volume fraction of mineral, i.e., $V_{m}=$ $45 \%$ for bone and $V_{m}=95 \%$ for nacre. For small aspect ratio, the buckling strength was small because the displacement of mineral mainly comes from rotation without bending, and the resistance to the rotation mainly originates from the shear deformation of protein matrix, as shown in Fig. 6. With the increase of the aspect ratio, the buckling displacement involves more bending of the mineral as well as tension of protein matrix. But when the aspect ratio becomes larger than a critical value, the buckling strength reaches a peak value, and further increase of the aspect ratio induces the decreases of buckling strength due to the reduction of both bending of mineral and tension deformation in protein.

It is interesting to compare the buckling behaviors of the staggered nanostructure with that of the continuous-fiber reinforced composite. Imagining each two neighboring mineral crystals of the staggered nanostructure in the $Z$ direction were welded together at the tip zone, the "imperfect" structure will then become ideal continuous structure. Its buckling strength is given by the Rosen model [19]

$$
\bar{\sigma}_{c}^{U}=\frac{1}{2\left(1-V_{m}\right)\left(1+\nu_{p}\right)}+\frac{\pi^{2} k V_{m}}{3 \rho^{2}}
$$

which sets the upper limit of buckling strength of staggered nanostructure of biological materials, as depicted in Fig. 7. When the aspect ratio became very large, the upper limit reduced down to the lower limit. Therefore, all the curves with different overlapping ratios $\lambda$ collapsed onto the upper/lower limits at extremely large aspect ratio.

The volume fraction of mineral also had significant effect on the buckling strength, which depends on the aspect ratio and overlapping ratio, as depicted in Fig. 8. The buckling strength increased with the volume fraction of mineral for different aspect ratios and overlapping ratios. When the aspect ratio was small $(\rho=5)$, the effect of volume fraction was significant, in particular at large overlapping ratio. When the aspect ratio became larger $(\rho=10,20)$, the effect of volume fraction was less significant as the rate of increase of the strength with the volume fraction became smaller. In addition, the buckling strength increased slowly when the volume fraction was small, but it increased faster abruptly when the volume fraction was large, in particular when $V_{m}>90 \%$. This might be the reason that hard biological materials often have very large volume fraction of mineral, such as sea shells.

Of note, in our previous work [17] the possible contact between the tips of adjacent mineral crystals was ignored. And, the buckling behaviors of the nanostructure were analyzed based on an RVE of a half length (i.e., $A_{\mathrm{I}}^{\prime} B_{\mathrm{II}}^{\prime} G_{\mathrm{I}}^{\prime} H_{\text {II }}^{\prime}$ ) of the present model $\left(A_{\mathrm{I}}^{\prime} B_{\text {IV }}^{\prime} G_{\mathrm{I}}^{\prime} H_{\mathrm{IV}}^{\prime}\right)$, as shown in Fig. $2(b)$. In addition, the mineral crystals were assumed in a staggering with a half overlapping length $(\lambda=1 / 2)$. In order to examine the effect of contact, here we make a comparison between the predictions of these two models. As in Ref. [17], we also adopt $A_{\mathrm{I}}^{\prime} B_{\mathrm{II}}^{\prime} G_{\mathrm{I}}^{\prime} H_{\mathrm{II}}^{\prime}$ as the RVE with 
(a)

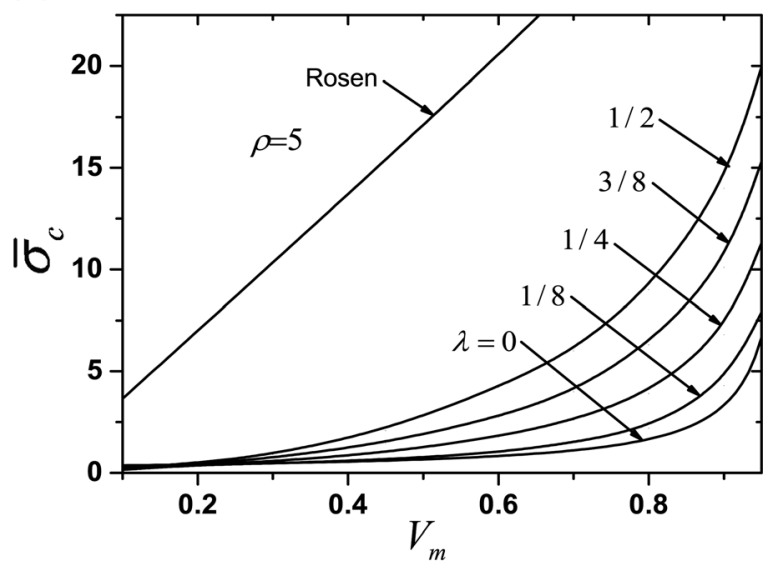

(b)

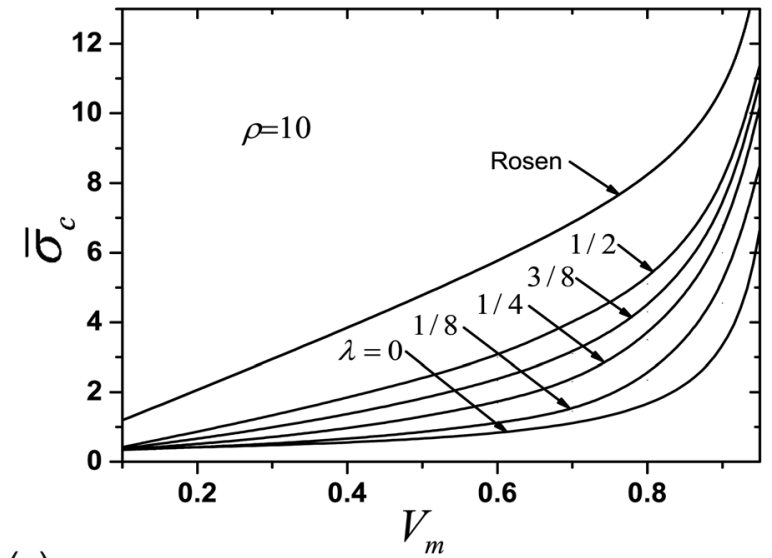

(c)

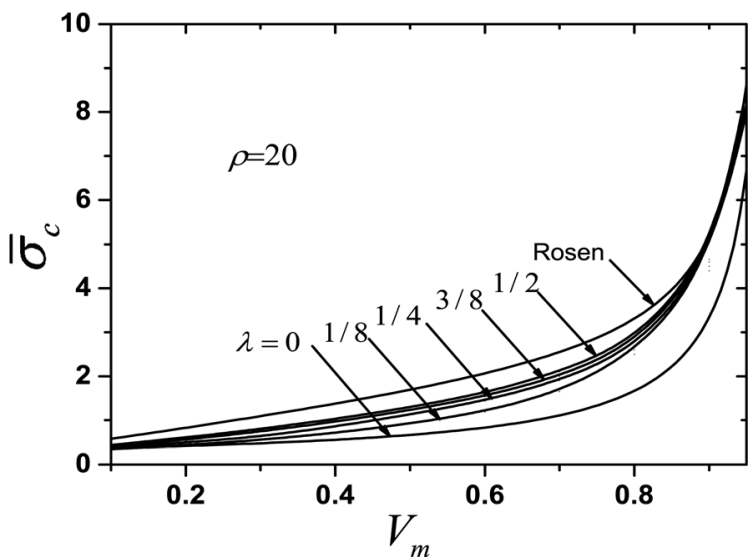

Fig. 8 The effect of volume fraction of mineral crystals on the buckling strength for (a) $\rho=5,(b) \rho=10$, and $(c) \rho=20$

$\lambda=1 / 2$. The difference is that the tips of adjacent mineral are connected via pinned joints in this study, while they were free in Ref. [17]. As we can see from Figs. 9 and 10, for the symmetric buckling, the predictions of the buckling strength and modes by the two models were similar. However, for the antisymmetric buckling, the predictions of the two models were different, in particular at small aspect ratio. The strength of the tip-pinned model of the present study was significantly larger than that of the tipfree model in the previous study. And their difference in buckling strength decreased with the increase of aspect ratio. This result can be understood from their difference in the buckling modes (a)

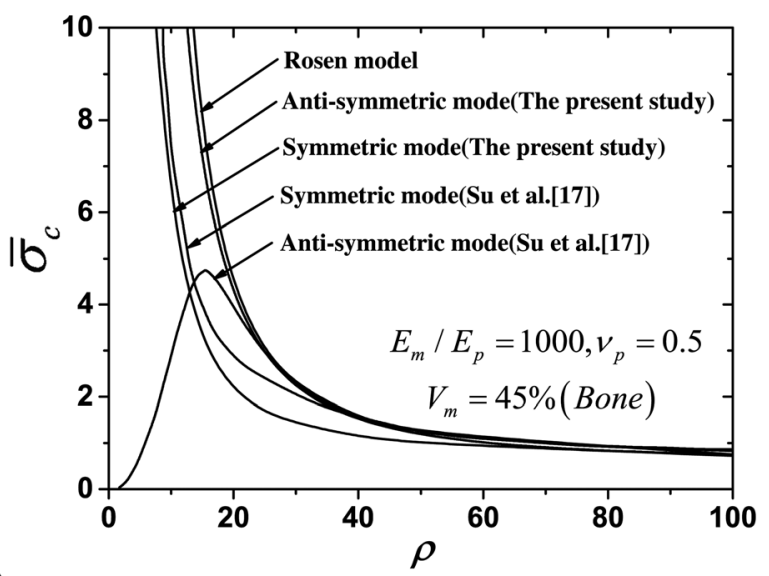

(b)

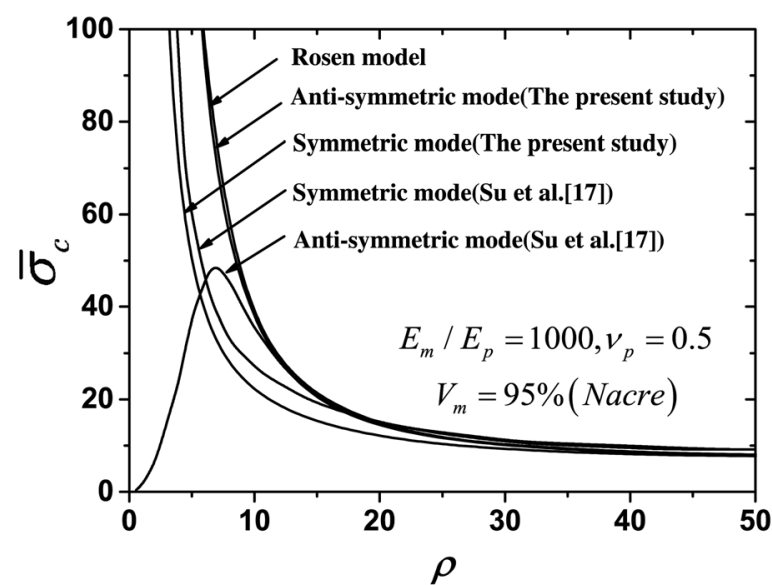

Fig. 9 The buckling strength predicted by the present study with tip-pinned model in comparison with that of previous study with tip-free model at various aspect ratios and volume fraction: (a) $V_{m}=45 \%$ for bone and (b) $V_{m}=95 \%$ for nacre

$\begin{array}{lcc}V_{m}=45 \% & \text { The present study } & \text { Su et al.[17] } \\ v_{p}=0.5 & \text { Symmetric Anti-Symmetric } & \text { Symmetric Anti-Symmetric }\end{array}$

Fig. 10 The buckling modes predicted by the present study with tip-pinned model and in comparison with that of previous study with tip-free model at various aspect ratios

(Fig. 10). The contact (modeled by pinned joint) restrained the rigid-body rotation of mineral, which shows that the contact between mineral tips may have significant effect on the buckling strength of nanostructure at small aspect ratio. Of note is that the 
actual interaction between the mineral tips should be between two extremities - a tip-pinned like in this study and tip-free like in previous works. Therefore, the actual buckling strength of biological materials should also be between the strength of the two extremities.

\section{Conclusions}

In this paper, a mechanical model, considering the overlapping ratio between mineral crystals and their contact at the tips, is proposed for studying the buckling behaviors of staggered nanostructure of biological materials. The effects of overlapping ratio, aspect ratio, and volume fraction of mineral crystals on the buckling mode and strength were analyzed systematically. Our main findings are summarized as follows:

(1) The overlapping ratio takes crucial roles in the buckling strength of the nanostructure of biological materials. The buckling strength increases with the overlap ratio in the range of $\lambda \in[0,1 / 2]$, and it reaches a peak value at $\lambda=1 / 2$. The mechanism is that the staggered arrangement with large overlapping ratio is able to enhance the buckling strength by restraining the rotation and bending of mineral crystals.

(2) The effect of overlapping ratio on the buckling strength depends on the aspect ratio and volume fraction of minerals. This effect is more significant at the small aspect ratio and large volume fraction.

(3) The contact between mineral tips may have significant effect on the buckling strength and buckling modes at small aspect ratio of minerals.

\section{Acknowledgment}

This work was supported by the National Natural Science Foundation of China (Grants Nos. 11025208, 11372042, 11532009 , and 11221202). Y.S. acknowledges the support from the Chinese Academy of Sciences via the "Hundred Talent Program" and support from NSFC (No. 11572323).

\section{Appendix: Derivation of the Shear Force on the Cross Section of Mineral}

According to the illustration in Fig. 4(b), the equilibrium equation of bending of mineral crystal is derived as

$$
\frac{d m_{1}}{d Z}-t_{2}+q_{1}+\stackrel{\circ}{t_{3}} \frac{d u_{2}(Z)}{d Z}=0
$$

where $t_{2}$ is the shear force of the mineral. Substituting Eqs. (13) and (14) into Eq. (A1), with considering $t_{3}^{\circ}=P_{c}$ is a constant and Eqs. (4)-(7), (9), and (11) in the text, yields the shear force of the mineral crystals $A^{\prime} B^{\prime}$ and $C^{\prime} D^{\prime}$, respectively,

$$
\begin{aligned}
t_{2} A^{\prime} B^{\prime}= & -\frac{E_{m} H_{m}^{3}}{12} v_{1}^{\prime \prime \prime}(Z)+\frac{G_{p} H_{m}\left(H_{m}+H_{p}\right)}{2 H_{p}}\left[v_{1}^{\prime}(Z)+v_{2}^{\prime}(Z)\right] \\
& +P_{c} v_{1}^{\prime}(Z) \\
t_{2}{ }^{\prime} D^{\prime}= & -\frac{E_{m} H_{m}^{3}}{12} v_{2}^{\prime \prime \prime}(Z)+\frac{G_{p} H_{m}\left(H_{m}+H_{p}\right)}{2 H_{p}}\left[v_{1}^{\prime}(Z)+v_{2}^{\prime}(Z)\right] \\
& +P_{c} v_{2}^{\prime}(Z)
\end{aligned}
$$

To consider the shear stress in protein matrix in the continuity conditions, such as at the cross section $A C, B D, C G$, and $F H$ of matrix, the shear stress in matrix is "attached" to the shear force of the mineral crystals as

$$
\begin{aligned}
Q^{A^{\prime}}= & t_{2}^{A^{\prime}}+\frac{1}{2}\left(Q^{A_{\mathrm{I}} C_{\mathrm{I}}}+Q^{E_{\mathrm{I}} G_{\mathrm{I}}}\right)=\frac{E_{m} H_{m}^{3}}{12 L^{3}} \\
& \times\left.\left[-v_{1}^{\prime \prime \prime}(\xi)+\frac{1}{2} h_{1} f_{1}^{\prime}(\xi)+\frac{1}{2} h_{2} g_{1}^{\prime}(\xi)\right]\right|_{\xi=0} \\
Q^{B^{\prime}}= & t_{2}^{B^{\prime}}+\frac{1}{2}\left(Q^{B_{\mathrm{I}} D_{\mathrm{I}}}+Q^{F_{\mathrm{I}} H_{\mathrm{I}}}\right)=\frac{E_{m} H_{m}^{3}}{12 L^{3}} \\
& \times\left.\left[-v_{1}^{\prime \prime \prime}(\xi)+\frac{1}{2} h_{1} f_{1}^{\prime}(\xi)+\frac{1}{2} h_{2} g_{1}^{\prime}(\xi)\right]\right|_{\xi=\lambda} \\
Q^{C^{\prime}}= & t_{2}^{C^{\prime}}+\frac{1}{2}\left(Q^{A_{\mathrm{I}} C_{\mathrm{I}}}+Q^{E_{\mathrm{I}} G_{\mathrm{I}}}\right)=\frac{E_{m} H_{m}^{3}}{12 L^{3}} \\
& \times\left.\left[-v_{2}^{\prime \prime \prime}(\xi)+\frac{1}{2} h_{1} f_{1}^{\prime}(\xi)-\frac{1}{2} h_{2} g_{1}^{\prime}(\xi)\right]\right|_{\xi=0} \\
Q^{D^{\prime}}= & t_{2}^{D^{\prime}}+\frac{1}{2}\left(Q^{B_{\mathrm{I}} D_{\mathrm{I}}}+Q^{F_{\mathrm{I}} H_{\mathrm{I}}}\right)=\frac{E_{m} H_{m}^{3}}{12 L^{3}} \\
& \times\left.\left[-v_{2}^{\prime \prime \prime}(\xi)+\frac{1}{2} h_{1} f_{1}^{\prime}(\xi)-\frac{1}{2} h_{2} g_{1}^{\prime}(\xi)\right]\right|_{\xi=\lambda}
\end{aligned}
$$

where $\quad h_{1}=\left(12 \rho^{2} / k\right)\left\{1 /\left[2 V_{m}\left(1-V_{m}\right)\left(1+v_{p}\right)\right]-\bar{\sigma}_{c} / V_{m}\right\}$; $h_{2}=-12 \rho^{2} \bar{\sigma}_{c} /\left(k V_{m}\right)$; and $Q^{A_{\mathrm{I}} C_{\mathrm{I}}}, Q^{B_{\mathrm{I}} D_{\mathrm{I}}}, Q^{E_{\mathrm{I}} G_{\mathrm{I}}}$, and $Q^{F_{\mathrm{I}} H_{\mathrm{I}}}$ are the shear force of matrix, i.e., the integral of the shear stress on the boundary $A_{\mathrm{I}} C_{\mathrm{I}}, B_{\mathrm{I}} D_{\mathrm{I}}, E_{\mathrm{I}} G_{\mathrm{I}}$, and $F_{\mathrm{I}} H_{\mathrm{I}}$, respectively.

\section{References}

[1] Currey, J., 2003, “The Many Adaptations of Bone," J. Biomech., 36(10), pp. 1487-1495.

[2] Ji, B., and Gao, H., 2010, "Mechanical Principles of Biological Nanocomposites," Ann. Rev. Mater. Res., 40(1), pp. 77-100.

[3] Espinosa, H. D., Rim, J. E., Barthelat, F., and Buehler, M. J., 2009, "Merger of Structure and Material in Nacre and Bone-Perspectives on de Novo Biomimetic Materials," Prog. Mater. Sci., 54(8), pp. 1059-1100.

[4] Currey, J., 1977, "Mechanical Properties of Mother of Pearl in Tension," Proc. R. Soc. London Ser. B, 196(1125), pp. 443-463.

[5] Fung, Y.-C., 1990, Biomechanics, Springer, New York.

[6] Okumura, K., and De Gennes, P.-G., 2001, "Why is Nacre Strong? Elastic Theory and Fracture Mechanics for Biocomposites With Stratified Structures," Eur. Phys. J. E, 4(1), pp. 121-127.

[7] Ji, B., and Gao, H., 2004, "Mechanical Properties of Nanostructure of Biological Materials," J. Mech. Phys. Solids, 52(9), pp. 1963-1990.

[8] Xie, Z., and Yao, H., 2014, "Crack Deflection and Flaw Tolerance in 'Brickand-Mortar' Structured Composites,” ASME Int. J. Appl. Mech., 6(2), p. 1450017.

[9] Qwamizadeh, M., Zhang, Z., Zhou, K., and Zhang, Y. W., 2015, “On the Relationship Between the Dynamic Behavior and Nanoscale Staggered Structure of the Bone," J. Mech. Phys. Solids, 78, pp. 17-31.

[10] Gao, H., Ji, B., Buehler, M. J., and Yao, H., 2004, "Flaw Tolerant Bulk and Surface Nanostructures of Biological Systems," Mech. Chem. Biosyst., 1(1), pp. 37-52.

[11] Gao, H., Ji, B., Jäger, I. L., Arzt, E., and Fratzl, P., 2003, "Materials Become Insensitive to Flaws at Nanoscale: Lessons From Nature," Proc. Natl. Acad. Sci., 100(10), pp. 5597-5600.

[12] Jäger, I., and Fratzl, P., 2000, "Mineralized Collagen Fibrils: A Mechanical Model With a Staggered Arrangement of Mineral Particles," Biophys. J., 79(4), pp. 1737-1746.

[13] Ji, B., and Gao, H., 2004, "A Study of Fracture Mechanisms in Biological Nano-Composites Via the Virtual Internal Bond Model," Mater. Sci. Eng. A, 366(1), pp. 96-103.

[14] Shao, Y., Zhao, H.-P., Feng, X.-Q., and Gao, H., 2012, "Discontinuous Crack-Bridging Model for Fracture Toughness Analysis of Nacre," J. Mech. Phys. Solids, 60(8), pp. 1400-1419.

[15] Zhang, Z., Liu, B., Huang, Y., Hwang, K., and Gao, H., 2010, "Mechanical Properties of Unidirectional Nanocomposites With Non-Uniformly or Randomly Staggered Platelet Distribution,” J. Mech. Phys. Solids, 58(10), pp. 1646-1660.

[16] Ji, B., Gao, H., and Jimmy Hsia, K., 2004, "How do Slender Mineral Crystals Resist Buckling in Biological Materials?" Phil. Mag. Lett., 84(10), pp. 631-641.

[17] Su, Y., Ji, B., Hwang, K.-C., and Huang, Y., 2012, "Micro-Buckling in the Nanocomposite Structure of Biological Materials," J. Mech. Phys. Solids, 60(10), pp. 1771-1790.

[18] Parnes, R., and Chiskis, A., 2002, "Buckling of Nano-Fibre Reinforced Composites: A Re-Examination of Elastic Buckling," J. Mech. Phys. Solids, 50(4), pp. 855-879.

[19] Rosen, B. W., 1965, "Mechanics of Composite Strengthening," Fiber Composite Materials, American Society of Metals, Cleveland, OH, pp. 37-75.

[20] Su, Y., Wu, J., Fan, Z., Hwang, K.-C., Song, J., Huang, Y., and Rogers, J. A., 2012, "Postbuckling Analysis and Its Application to Stretchable Electronics," J. Mech. Phys. Solids, 60(3), pp. 487-508. 